\title{
Flapping of heavy inverted flags: a fluidelastic instability
}

\author{
Mohammad Tavallaeinejad ${ }^{1}$, Michael P. Païdoussis ${ }^{1}$, Manuel Flores Salinas ${ }^{2}$, Mathias Legrand ${ }^{1}$, \\ Mojtaba Kheiri ${ }^{3}$, and Ruxandra M. Botez ${ }^{2}$ \\ ${ }^{1}$ Department of Mechanical Engineering, McGill University, Montréal, Québec, Canada \\ ${ }^{2}$ Laboratoire de recherche en commande active, avionique et aéroservoélasticité, École de Technologie Supérieure, Montréal, \\ Québec, Canada \\ ${ }^{3}$ Department of Mechanical, Industrial and Aerospace Engineering, Concordia University, Montréal, Québec, Canada
}

\begin{abstract}
Wind tunnel experiments are described in this paper, aiming to examine the global dynamics of heavy inverted flags, with a specific focus on the underlying mechanism of large-amplitude flapping, which occurs at sufficiently high flow velocities. This problem is of interest because no consensus exists as to the mechanism, specifically whether it is a vortex-induced vibration or a self-excited vibration - the answer being not only of fundamental interest, but also important for energy harvesting applications.

The effect of vortex shedding from both leading and trailing edges was investigated via experiments with flags modified by serrations to the leading edge and a long rigid splitter plate at the trailing edge, so as to disrupt leading- and trailing-edge vortices and to inhibit interactions between counter-rotating leading-edge vortices, if they exist. The relatively small quantitative changes in the critical flow velocity, amplitude and frequency of oscillations, as well as the near-identical qualitative behaviour, of plain and modified flags suggests that the global qualitative dynamics of heavy inverted flags is independent of vortex shedding from the leading and trailing edges; i.e., periodic vortex shedding is not the cause but an effect of large-amplitude flapping.

Additional experiments showed that the dominant frequencies of flapping and the lift force on the flag are generally not synchronised, and multiple frequencies occur in the lift signal, reinforcing the conclusion that vortex shedding is not the cause of flapping.

Our experimental results suggest that self-excited vibration through a fluidelastic instability, i.e. flutter, is the underlying mechanism for the flapping of heavy inverted flags.
\end{abstract}

\section{Introduction and background}

The present work investigates the flow-induced vibration of a flexible thin plate in axial flow: a cantilevered thin plate (or 'flag') of length $L$, height $H$ and thickness $h$ subjected to a fluid flowing axially with velocity $U$ and directed from the free end towards the clamped one, otherwise known as an "inverted flag" as shown in Figure 1 [5]. The dynamics of inverted flags is governed by four dimensionless parameters: (i) mass ratio $\mu=\rho_{\mathrm{f}} L / \rho_{\mathrm{p}} h, \rho_{\mathrm{f}}$ and $\rho_{\mathrm{p}}$ being the mass density of the fluid and plate, respectively; (ii) aspect ratio $R=H / L$, (iii) Reynolds number $R e_{L}=U L / v, v$ being the flow kinematic viscosity; and (iv) dimensionless flow velocity $\Pi=\sqrt{\rho_{\mathrm{f}} H L / D} L U, D$ being the flexural rigidity of the flag.

The sequence of the dynamical states displayed by an inverted flag as the flow velocity is increased from zero, as observed experimentally/theoretically by different researchers, is: stretched-straight [5] $\rightarrow$ buckled with small deflections $[13,16] \rightarrow$ small-amplitude asymmetric deformed flapping $[2,13,16]$ $\rightarrow$ large-amplitude flapping [5] $\rightarrow$ chaotic $[11,13] \rightarrow$ fully deflected $[2,5] \rightarrow$ flipped flapping with the free-end pointing downstream $[2,13]$.

Sader et al. [11], using analytical modelling and experimental measurement, proposed for the first time that the large-amplitude flapping motion of inverted flags is a vortex-induced vibration (VIV). The flapping phenomenon was attributed to the periodic formation and synchronized shedding of vortices, which is a characteristic of VIV, from the trailing and leading edges. The VIV mechanism also appeared to be able to successfully explain other aspects of the dynamics of inverted flags under heavy fluid loading, i.e. $\mu \gtrsim O(1)$. Some examples are the Strouhal number independence from the Reynolds number and the flapping occurrence above a minimum amplitude. 


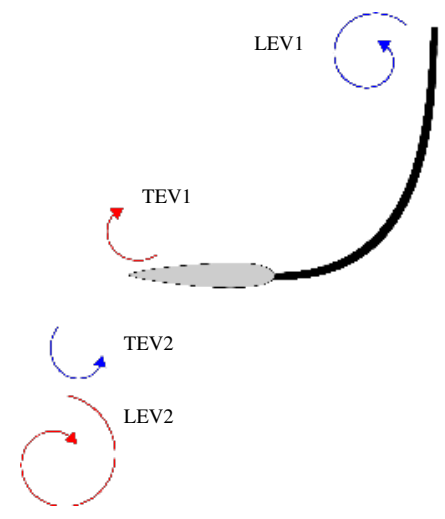

Figure 1: Inverted flag in the large-amplitude flapping regime with shedding leading-edge vortices (LEVs) and trailing-edge vortices (TEVs) downstream.

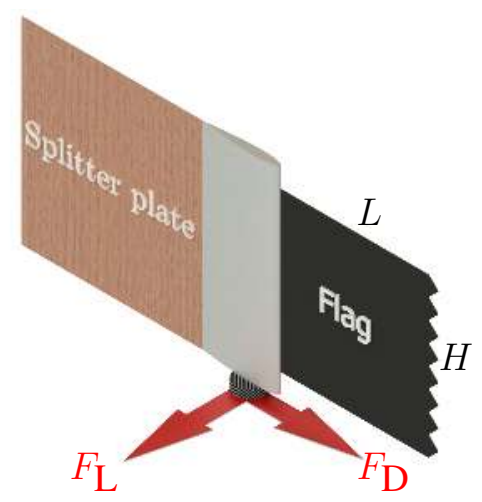

Figure 2: Experimental set-up for a serrated inverted flag with a rigid splitter plate where the forces at the flagpole are measured by a force balance.

However, Sader et al. [11] performed a scaling analysis and predicted that VIV cannot occur for $\mu \ll 1$, even though large-amplitude flapping does. In addition, some studies published later showed that inverted flags can perform large-amplitude flapping even under circumstances where regular vortex formation and shedding from leading- and trailing-edges are either completely absent or weakened. For example, in an experimental study, Pazhani [9] investigated the effect of leading-edge serrations, where they found that the serrated flag does display large-amplitude flapping, even though the regular vortex formation and shedding from the leading edge was disrupted. Gurugubelli and Jaiman [3] performed direct numerical simulations for an inverted flag $(\mu=1)$ in the $R e_{L}=3 \times 10^{4}$ flow regime. They showed that the frequency of dominant vortex-induced forces may not be synchronised with the frequency of the transverse flapping motion. Goza et al. [1] showed computationally that for sufficiently heavy (or small mass ratios) flags, i.e. $\mu \ll 1$, large-amplitude flapping occurs even for $R e_{L}<50$, where vortex shedding essentially does not occur; hence, neither large-amplitude nor small-amplitude flapping can be attributed to the classical VIV mechanism. [3] performed simulations in which a long rigid splitter plate was attached to the flag trailing edge, thus delaying trailing-edge vortex formation and shedding to large distances from the flag and also eliminating the interactions between vortices detached from the leading edge at the cycle extremities They found that the flags undergo large-amplitude flapping regardless $-\mathrm{a}$ prediction not yet verified experimentally, which is, in fact, one of the objectives of the present paper.

Although the above-referenced studies showed that VIV is not always the underlying mechanism for the large-amplitude flapping of inverted flags, they did not put forward an alternative physical explanation. Motivated by this gap in knowledge, Tavallaeinejad $[14,15]$ developed mathematical models for small-aspect-ratio and two-dimensional heavy inverted flags, i.e. $\mu \ll 1$, respectively, and showed that a plausible explanation for large-amplitude flapping of heavy inverted flags is self-excited vibration emanating from a fluidelastic instability. The model in [15] was able to explain several aspects of the dynamics of heavy inverted flags observed experimentally or predicted computationally, such as the onset and sequence of instabilities, based on principles used for developing governing equations for similar fluid-structure systems, such as flexible wings and pipes conveying fluid, which are known to be subject to fluidelastic instabilities.

The primary purpose of this paper, as also suggested by the title, is to explore experimentally the underlying mechanism for large-amplitude flapping of heavy inverted flags. The correlation between vortex shedding and the flapping mechanism, and more specifically, the qualitative and quantitative effects of disruption of both leading-edge vortices (LEVs) and trailing-edge vortices (TEVs) on the onset, frequency and amplitude of flapping are examined. In the present experiments, flags of mass ratio $\mu \in\left[\begin{array}{ll}0.07 & 0.21\end{array}\right]$ have been tested in the flow regime $\operatorname{Re}_{L} \in\left[\begin{array}{l}3.4 \times 10^{4} 2 \times 10^{5}\end{array}\right]$.

The rest of the paper is organized as follows. First, experiments with a rigid splitter plate attached to the trailing-edge of the inverted flag are described in $\$ 2.1$, aiming to evaluate the importance of the existence of TEVs and to examine the effects of forced disconnection between counter-rotating LEVs on large-amplitude flapping. Second, experiments with inverted flags with a serrated leading-edge, similar to 
those used in [9], and with a splitter plate at the trailing edge are described in §2.2 (see Figure 2); these experiments explore the effects of simultaneous disruption of LEVs and TEVs. Finally, the synchronization of lift and displacement and phase dynamics on plain heavy inverted flags are studied in §2.3.

\section{Results and discussion}

\subsection{Dynamics of inverted flags with a rigid splitter plate attached to the trailing edge}

The experiments were conducted in a subsonic wind tunnel with a fairly large test-section and low $(<1 \%)$ turbulence intensity. The flow velocity in the test-section was incremented in small steps $(\approx 0.5 \mathrm{~m} / \mathrm{s})$ and the flag motion was recorded via a high-speed camera ( $80-280 \mathrm{fps}$ ) for each velocity. An image processing technique was then utilized to extract the time history of oscillations (for more details, refer to [13]). Brass and polycarbonate plates, i.e. 'flags', $(0.5 \leq R \leq 4.0$ and $0.07 \leq \mu \leq 0.20)$ were used in
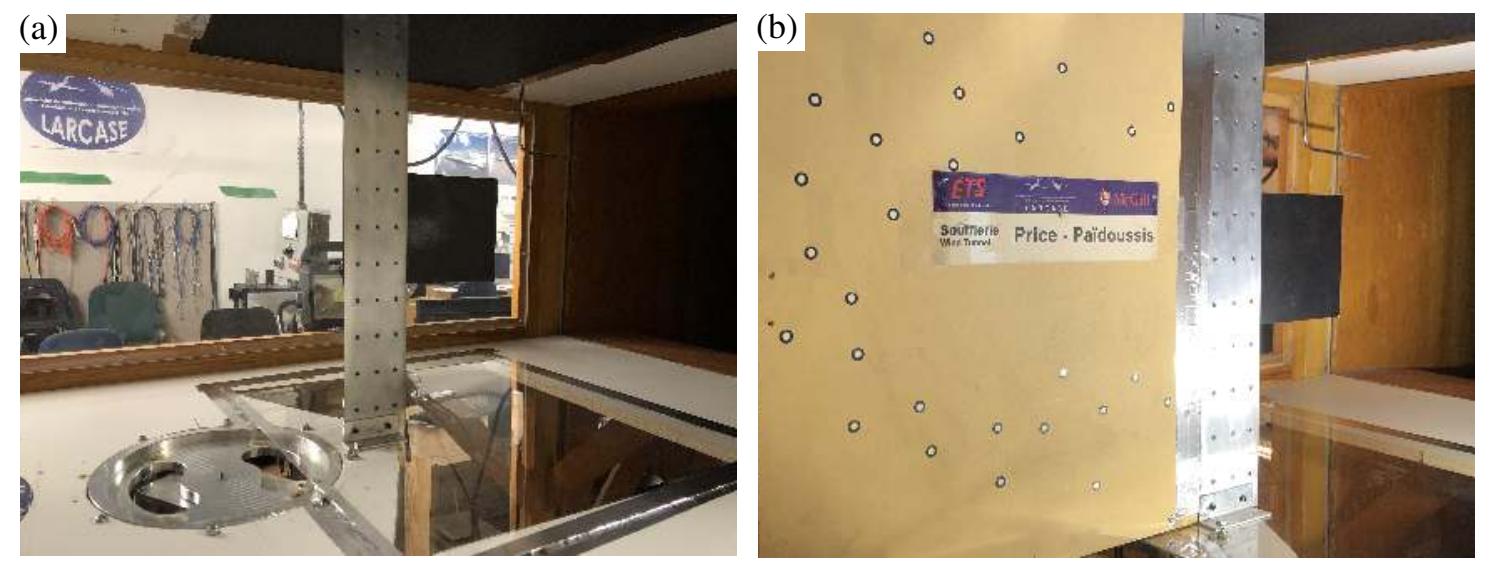

Figure 3: Experimental set-up for the inverted flag (a) without and (b) with the rigid splitter plate.

the experiments conducted with and without a rigid splitter plate; see Table 1 for flag dimensions and other properties. The splitter plate was made from a plywood sheet of thickness $h_{\mathrm{s}}=10 \mathrm{~mm}$, height

\begin{tabular}{cccccc}
\hline Flag & Material & $L \times H$ & $h$ & $R$ & $\mu$ \\
\hline A & Polycarbonate & $150 \times 225$ & 1.02 & 1.5 & 0.15 \\
B & Polycarbonate & $150 \times 600$ & 0.76 & 4.0 & 0.20 \\
C & Polycarbonate & $160 \times 160$ & 0.76 & 1.0 & 0.20 \\
D & Polycarbonate & $160 \times 160$ & 1.02 & 1.0 & 0.16 \\
E & Brass & $198 \times 101$ & 0.38 & 0.5 & 0.07 \\
\hline
\end{tabular}

Table 1: Labels and dimensions of inverted flags tested in experiments with and without rigid splitter plate.

Dimensions are in $\mathrm{mm}$.

$H_{\mathrm{s}}=610 \mathrm{~mm}$, and length $L_{\mathrm{s}}=1800 \mathrm{~mm}$, and was secured firmly to the walls of the test section, as pictured in Figure 3(b); no significant motion of the splitter plate was observed during the experiment, even at very high flow velocities.

Figure 4(a) shows bifurcation diagrams for the tip rotation of flag C (circles) and flag D (diamonds) with the rigid splitter plate (filled symbols) and without it (empty symbols). As shown, with increasing the flow velocity, the flag reaches a critical point, beyond which it abruptly starts performing large-amplitude symmetric flapping around the position of rest; for example, for flag $\mathrm{C}$, the dimensionless critical flow velocities (when sweeping the flow velocity up) are $\Pi_{f}^{i}=1.77$ and $\Pi_{f}^{i}=1.69$, with and without the splitter plate, respectively. Figure 4(a) also shows that the critical flow velocities when sweeping up (black markers) and down (red markers) are different, i.e. flapping emanates from a subcritical Hopf bifurcation. As seen, flag D exhibits a stronger subcritical behaviour and larger hysteresis loop, compared to flag C. The flapping amplitude increases with the flow velocity, while, as seen in Figure 4(b), the dimensionless flapping frequency, $\bar{f}$, slightly increases first, but then decreases with flow velocity. A slight reduction 

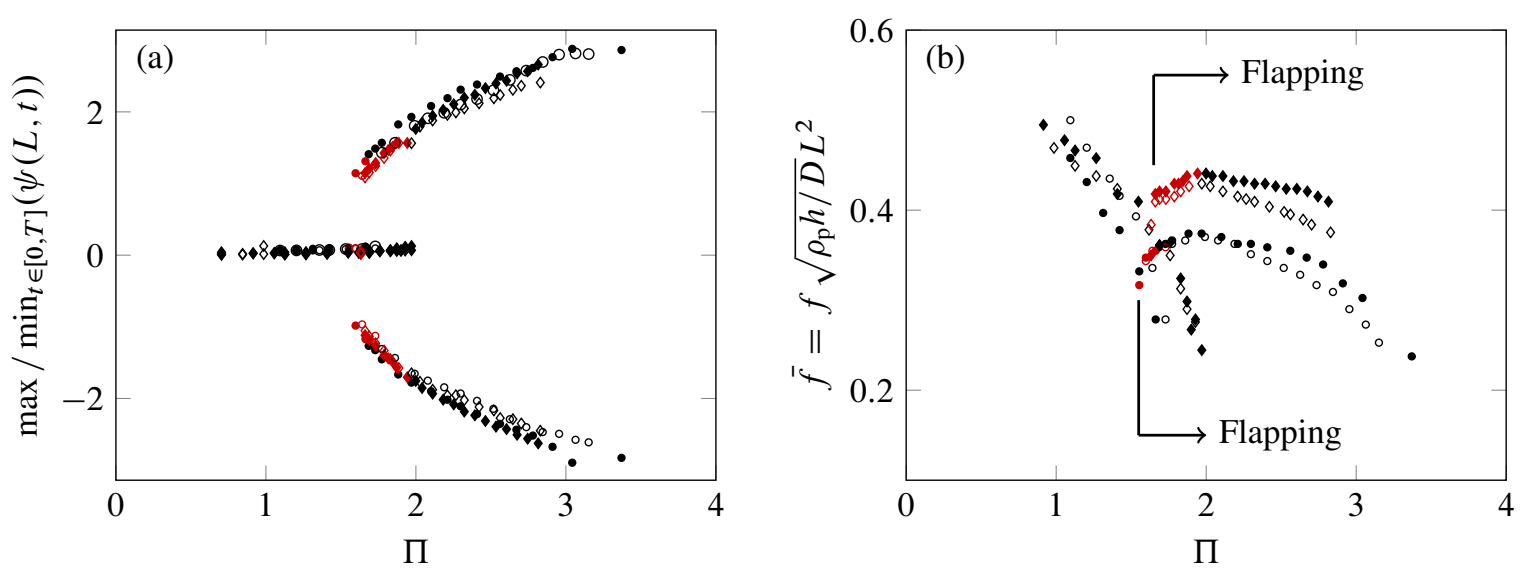

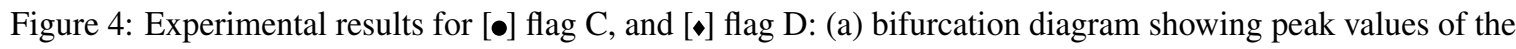
rotation angle of the free-end of the flag as a function of the dimensionless flow velocity, and (b) dimensionless dominant frequency of oscillation as a function of the dimensionless flow velocity. Empty and filled markers correspond to the flag dynamics with and without the splitter plate, respectively. Black and red markers correspond to the flow velocity sweep up and down, respectively.

is noted for the flapping amplitude of the flag with a splitter plate. Similar observations were made by Gurugubelli and Jaiman [3] who performed simulations on two-dimensional flags with $\mu=1$ in the intermediate-Reynolds-number flow regime $\left(\operatorname{Re}_{L}=3 \times 10^{4}\right)$. They observed that the inverted flag with a splitter plate exhibits only two counter-rotating vortices shed from the leading edge over the flapping cycle. Through a systematic analysis of vorticity distribution and the coupled flag dynamics, they showed that the absence of trailing-edge vortices (or more precisely, their displacement farther away from the flag), and the inhibition of vortex-vortex interaction lead to a larger pressure distribution at the trailing edge and to a slightly smaller drag at the leading edge. This would then result in a smaller bending moment, which in turn leads to a reduction in the curvature along the flag.

As seen in Figure 4(b), the frequency of oscillation is also reduced slightly when the splitter plate is introduced. For instance, the maximum reduction in flapping frequency for flag $\mathrm{D}$ is at $\Pi \simeq 2.8$, where the frequency is reduced by almost $8 \%$. This may also be associated with the absence of trailing edge vortices in close proximity to the flag, caused by the rigid splitter plate. Using the computational results and observations provided by Gurugubelli and Jaiman [3], we hypothesise that, when there is no splitter plate, the trailing-edge vortex formation and shedding accelerates the reduction in the pressure distribution over the flag, which consequently leads to a faster transition of maximum deflection from one side to the other, hence to a higher frequency. Such a hypothesis needs to be verified through careful flow visualization and measurements.

Figure 5 shows that the onset of large-amplitude flapping for all flags tested (except for flag B) is delayed when the splitter plate is added. This may be linked to the pressure reduction close to the

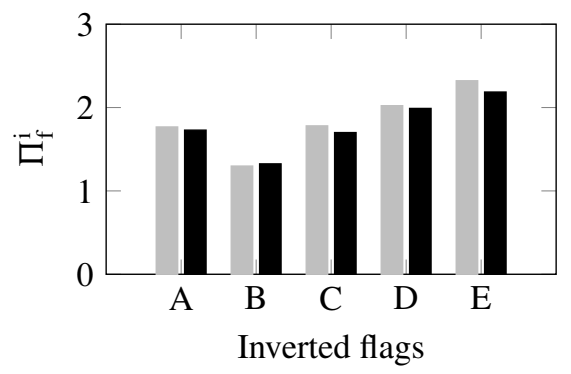

Figure 5: Dimensionless critical flow velocity for the onset of large-amplitude flapping of different inverted flags when sweeping up the flow velocity; $[\square]$ with and $[\square]$ without the rigid splitter plate.

leading edge of the inverted flag due to the splitter plate: the presence of the splitter plate is speculated to introduce a small additional damping to the dynamical system. Consequently, the critical flow velocity for large-amplitude flapping increases and the flapping amplitude and frequency become smaller at the onset 
of large-amplitude flapping.

However, all these differences are very slight. The main and most significant conclusion is that introducing a splitter plate has a minimal influence on the critical flow velocity for large-amplitude flapping, its amplitude and frequency. Since no PIV measurements were undertaken in the present experiments, no information on the size, magnitude and dynamics of LEVs and TEVs can be provided. Nevertheless, computational studies, for example [1, 2, 3, 10], as well as experimental studies [16], show that LEV are as strong as TEV. Since LEV are formed at the leading-edge, compared to TEV, it is expected that they have a much stronger effect on the fluid flow in the vicinity of the leading-edge and thus on the overall dynamics of the flag. This might then be considered as a plausible explanation as to why suppressing, or more precisely delaying, vortex shedding from the trailing-edge via a splitter plate does not change the dynamics significantly.

\subsection{Dynamics of inverted flags with leading-edge serrations and a splitter plate at the trailing edge}

In order to further understand the effects of vortex formation and shedding on the global dynamics of inverted flags, a serrated leading-edge geometry (chevron) with height $H_{\mathrm{s}}=10 \mathrm{~mm}$ and width $W_{\mathrm{s}}=10 \mathrm{~mm}$ was introduced to polycarbonate flags of $0.25 \leq R \leq 3$; see Figure 2 . Using flow visualization techniques, Pazhani [9] has shown that the serrations (as if they were miniaturized delta wings) produce small counter-rotating pairs of vortices which interact with the primary tip vortex and disrupt its formation and periodic shedding from the leading edge.

The experiments with serrated flags performed by Pazhani [9] have been repeated in the present study, with wider flags - to minimize the three-dimensionality of the flow caused by the side edges of the flag-also using a rigid splitter plate at the trailing edge of the flag to impede interactions among the separated shear layers. Mainly qualitative experiments were conducted. Insofar as the onset and amplitude of large-amplitude flapping is concerned, the responses are similar to those of plain inverted flags. For instance, the experimental results for flags of $R=3.0$ in Figure 6 show no notable differences in the critical values of flow velocity, as well as the amplitude and the frequency of oscillation, with and without the splitter plate and serrations. Our observations from the experiments with inverted flags modified by a
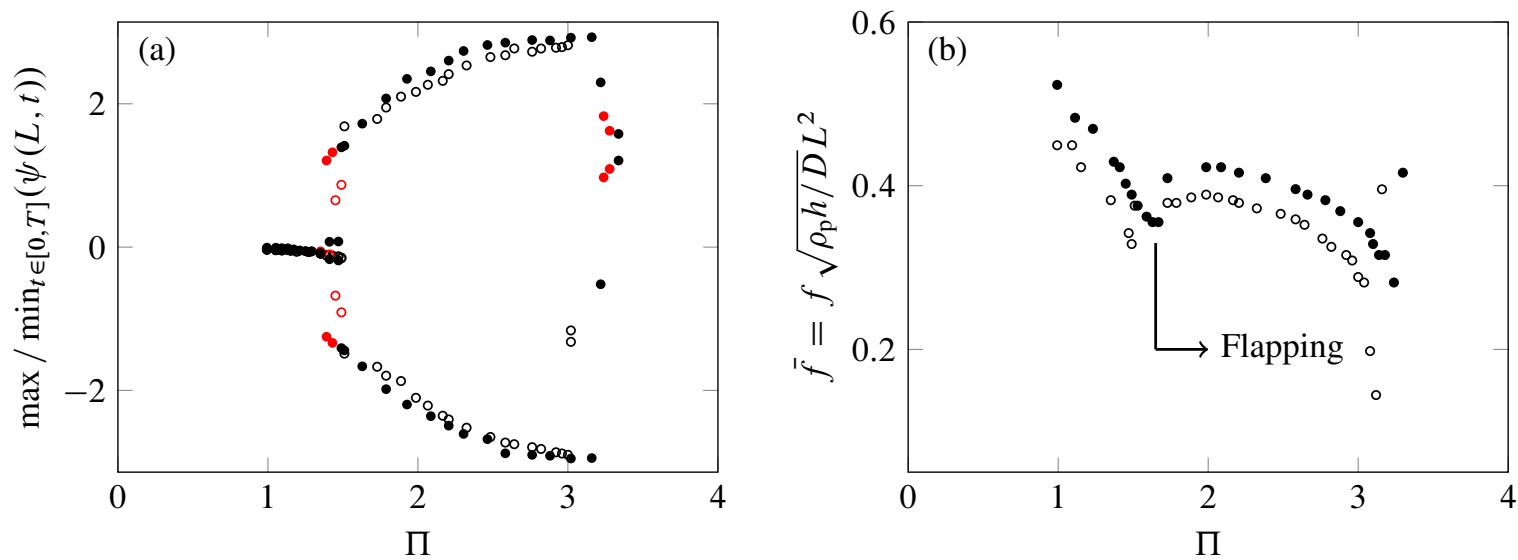

Figure 6: Experimental results for polycarbonate inverted flags of aspect ratio $R=3.0$ : (a) peak values of the rotation angle of the free-end of the flag, and (b) dimensionless dominant frequency of oscillations, as a function of the dimensionless flow velocity; [•] plain inverted flag and [o] serrated flag with splitter plate at the trailing edge. Black and red symbols correspond to flow velocity sweep up and down, respectively.

serrated leading edge and a splitter plate at the trailing edge confirm that the dynamical characteristics of heavy inverted flags are not very sensitive to: (i) the periodic formation and shedding of vortices from the leading and trailing edges, and (ii) vortex-vortex interactions (if any exist). Hence, a mechanism different from VIV should be at work for large-amplitude flapping of heavy inverted flags; self-excited vibration emerging from a fluidelastic instability is deemed to be a credible alternative able to explain many aspects of the dynamics of heavy inverted flags. This will be discussed further in $§ 2.3$. 


\subsection{Synchronization of lift and displacement and phase dynamics of heavy plain inverted flags}

Several experiments were conducted to measure simultaneously the forces acting on the flag (i.e. lift and drag) and its motion. The phase difference between the time traces of the fluid forces and flag displacements were examined. Moreover, the dominant frequencies of the fluid forces were obtained.

These experiments were motivated by an observation made by Goza et al. [1] for large-amplitude flapping of heavy inverted flags. They reported that, compared to light flags, several additional vortices are shed per cycle for heavy ones, resulting in additional peaks in the frequency spectrum of the lift signal. Moreover, the frequency associated with the highest peak in the lift spectrum was different from that of the displacement spectrum. This led Goza et al. [1] to refer to the large-amplitude flapping of massive flags as "not-classical VIV." An earlier similar observation was made by Gurugubelli and Jaiman [3] for an inverted flag with $\mu=1$.

In order to investigate experimentally the existence of synchronization between the lift force and displacement for heavy inverted flags, stainless-steel flags of varying length (hence, varying mass ratio), labelled S1 to S4, were tested (see Table 2). The transverse (lift) and streamwise (drag) components of the

\begin{tabular}{cccccc}
\hline Flag & $L(\mathrm{~mm})$ & $\mu$ & $R$ & $\Pi / U$ & $\sqrt{\rho_{\mathrm{f}} h / D} L^{2}$ \\
\hline S1 & 100 & 0.21 & 0.75 & 0.3653 & 0.0822 \\
S2 & 70 & 0.15 & 1.07 & 0.2140 & 0.0403 \\
S3 & 50 & 0.10 & 1.50 & 0.1292 & 0.0206 \\
S4 & 35 & 0.07 & 2.14 & 0.0756 & 0.0101 \\
\hline
\end{tabular}

Table 2: Stainless-steel inverted flags utilized in experiments; $H=75 \mathrm{~mm}$ and $h=0.08 \mathrm{~mm}$. The dimensionless frequency can be obtained as $f^{*}=f \sqrt{\rho_{\mathrm{f}} h / D} L^{2}$.

fluid flow force were measured simultaneously at the flagpole, utilizing an in-house built aerodynamic balance (Mini45-E Array Technology Incorporated Inc). Time traces of the lift, $F_{\mathrm{L}}(0, t)$, and the drag, $F_{\mathrm{D}}(0, t)$, components (see Figure 2) were collected at $1000 \mathrm{~Hz}$; the sampling rate for the tip transverse displacement, $w(L, t)$, was $160-280 \mathrm{~Hz}$ (see [13] for more details).

\subsubsection{Frequency characteristics}

Figure 7 shows the time trace and power spectral density (PSD) plots for the dimensionless tip transverse displacement, $w(L, t) / L$, and lift for flag $\mathrm{S} 1$ at different flow velocities: $(\mathrm{a}, \mathrm{b}) U=5.7 \mathrm{~m} / \mathrm{s}(\Pi=2.08)$, (c,d) $U=7.7 \mathrm{~m} / \mathrm{s}(\Pi=2.81)$, and $(\mathrm{e}, \mathrm{f}) U=8.3 \mathrm{~m} / \mathrm{s}(\Pi=3.03)$. In the PSD plots for the lift signal, the peaks are labelled sequentially as $f_{\mathrm{L} 1}, f_{\mathrm{L} 2}$ etc. from low to high frequencies. As seen in Figure 7(b,d), $f_{1}=f_{\mathrm{L} 1}$, where $f_{1}$ is the displacement dominant frequency. Also, from the lift PSD, $f_{\mathrm{L} 1}$ has several higher harmonics, such as $f_{\mathrm{L} 2}$ and $f_{\mathrm{L} 3}$, with comparable powers, while in the displacement PSD, only one predominant frequency harmonic exists, supporting the observations by Goza et al. [1] discussed above. By increasing the flow velocity, motion becomes chaotic-like at $U=8.3 \mathrm{~m} / \mathrm{s}(\Pi=3.03)$. As seen from the lift PSD, $f_{\mathrm{L} 2}$ becomes the dominant frequency, while still $f_{1}=f_{\mathrm{L} 1}$; the loss of $1: 1$ synchronization with departure from a periodic behaviour can also be seen in Figure 7(e,f). One may conclude from Figure 7(a-f) that by increasing the flow velocity, the dynamics of the flag and that of the flow become more complex leading eventually to chaotic-like motion. This is evidenced by the increase of irregularity in the time histories and the fact that the spectra show a broader range of frequencies with a distinct subdominant peak in the PSD of lift. The increase of decoherence between the flag displacement and lift, as the flow velocity is increased, should also be noted.

The spectrograms of the displacement and lift signals are presented in Figure $7(\mathrm{~g}, \mathrm{~h})$, respectively, showing an increase in the dynamic activity with increasing flow velocity. In particular, the lift spectrogram shows the presence of multiple frequencies with comparable powers, which are distinguishable for most of the flow velocity range. This suggests that the wake flow contains vortices of comparable strength, shed at different frequencies (including the flapping frequency), indicating that the vortex shedding does not fully control the flapping. Nevertheless, detailed studies of the flow dynamics, perhaps via PIV measurements, are required to validate what was discussed above; such experiments are deferred to future investigations. 

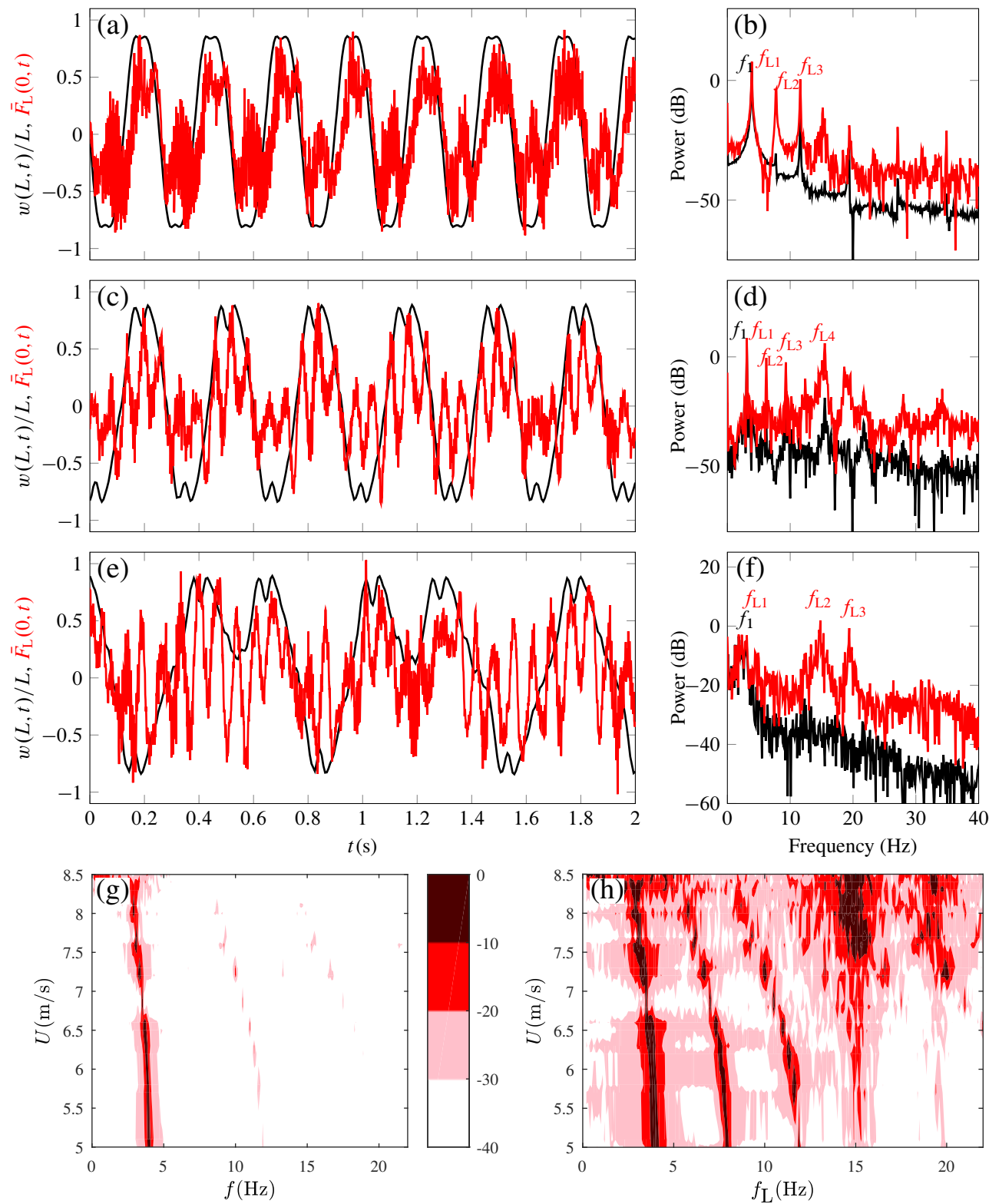

Figure 7: (a,c,e) Time traces, (b,d,f) PSDs and (g,h) spectrograms for flag S1: [- ] tip transverse displacement and $[-]$ normalized lift $\bar{F}_{\mathrm{L}} ;(\mathrm{a}, \mathrm{b}) U=5.7 \mathrm{~m} / \mathrm{s}(\Pi=2.08),(\mathrm{c}, \mathrm{d}) U=7.7 \mathrm{~m} / \mathrm{s}(\Pi=2.81)$, and $(\mathrm{e}, \mathrm{f})$ $U=8.3 \mathrm{~m} / \mathrm{s}(\Pi=3.03)$. Power spectrum in $\mathrm{dB}$.

Figure 8 shows the time trace and PSD plots for (a,b) flag S2, (c,d) flag S3, and (e,f) flag S4, respectively. Similarly to the discussion on Figure 7, the displacement PSDs show that the power is mostly centred around one frequency (i.e. $f_{1}$ ), while the lift PSDs show an almost evenly distributed power over multiple frequencies. For example, as shown in Figure 8(d) for flag S3, the dominant displacement frequency is $f_{1}=18 \mathrm{~Hz}$, while that for the lift is $f_{\mathrm{L} 3}=36 \mathrm{~Hz}$. There are also other peaks in the lift PSD, which occur at $f_{\mathrm{L} 1}=14.1 \mathrm{~Hz}, f_{\mathrm{L} 2}=f_{1}=18 \mathrm{~Hz}, f_{\mathrm{L} 4}=3 f_{1}=54 \mathrm{~Hz}$, and $f_{\mathrm{L} 5}=5 f_{1}=90 \mathrm{~Hz}$; however, no similarly strong peaks can be found in the displacement frequency content. This indicates that vortex shedding may be synchronized to a higher displacement harmonic; in the present case, the vortex shedding frequency is twice the flapping frequency, giving rise to a 1:2 synchronization. The difference between the dominant (peak) frequencies of the lift and flapping for flag S2 is illustrated further through spectrograms shown in Figure 8(g,h).

\subsubsection{Phase dynamics}

It is known that in the case of VIV-driven motion of a circular cylinder in cross-flow, as the flow velocity is varied, sharp changes occur in the phase difference between the fluid forces and cylinder motion at 

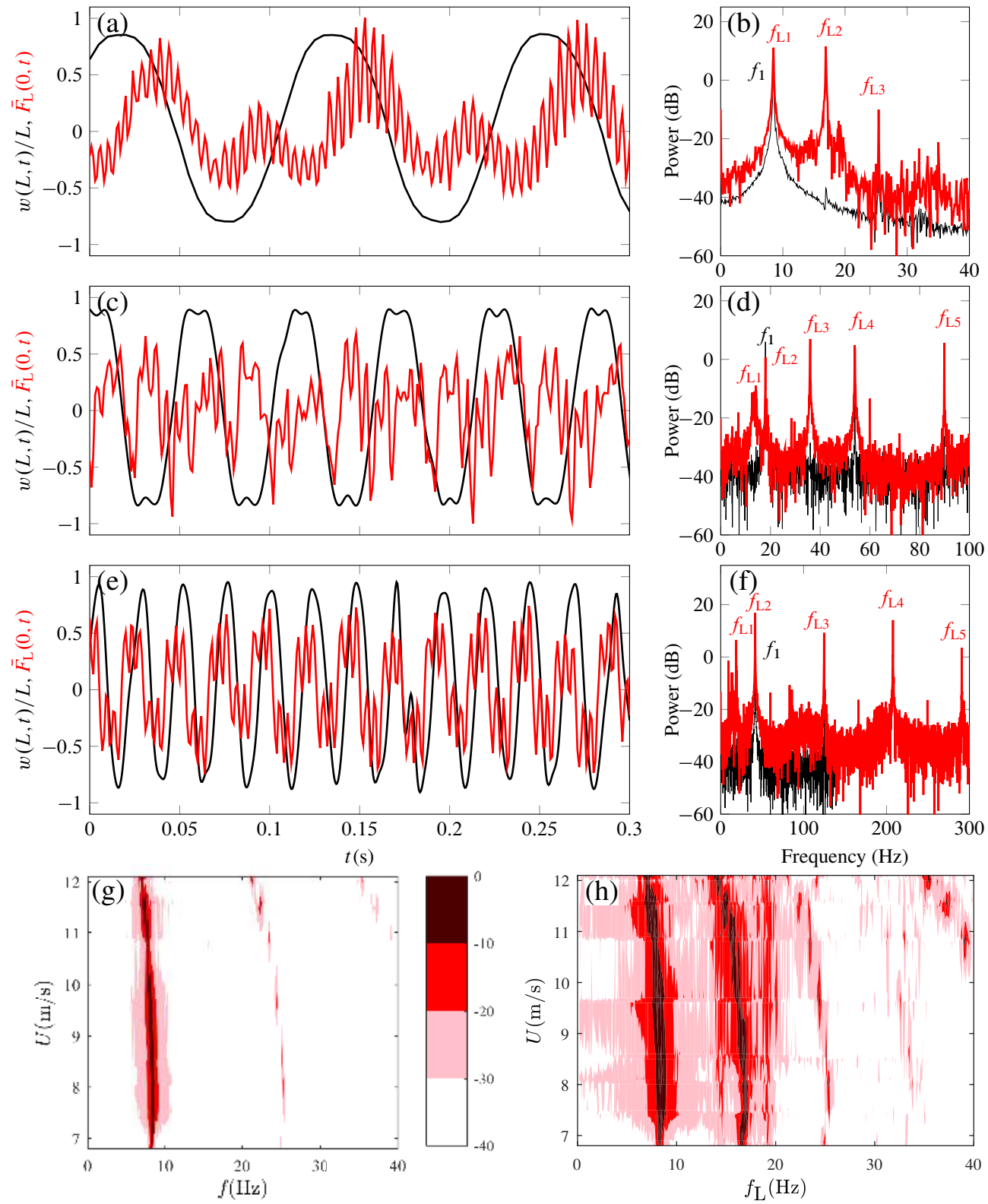

Figure 8: (a,c,e) Time trace and (b,d,f) PSD plots for flags S2 to S4, respectively: [-] tip transverse displacement and [-] normalized lift $\bar{F}_{\mathrm{L}} ;(\mathrm{a}, \mathrm{b}): U=7.4 \mathrm{~m} / \mathrm{s}(\Pi=1.58) ;(\mathrm{c}, \mathrm{d}): U=14.4 \mathrm{~m} / \mathrm{s}(\Pi=1.86)$; and $(\mathrm{e}, \mathrm{f}): U=19.0 \mathrm{~m} / \mathrm{s}(\Pi=1.44)$. (g,h) Spectrogram plots for flag S2. Power spectrum in $\mathrm{dB}$.

resonance. In particular, the phase between the cross-flow force (i.e. lift) and the transverse displacement of the cylinder jumps from nearly 0 to about $\pi$ (see $[4,12,17]$ ).

In this paper, the instantaneous phase difference between the time series obtained for the transverse displacement of the flag and the lift are calculated using the Hilbert transform $[4,6]$. The instantaneous phase is defined as $\phi_{\mathrm{w}}(t)=\operatorname{atan}[w(L, t) / \hat{w}(L, t)]$ and $\phi_{\mathrm{F}}(t)=\operatorname{atan}\left[F_{\mathrm{L}}(0, t) / \hat{F}_{\mathrm{L}}(0, t)\right]$, where $\hat{w}(L, t)$ and $\hat{F}_{\mathrm{L}}(0, t)$ are the Hilbert transforms of $w(L, t)$ and $F_{\mathrm{L}}(0, t)$, respectively. Next, the instantaneous phase difference, $\phi_{\mathrm{d}}(t)$, between lift and displacement is calculated as $\phi_{\mathrm{d}}(t)=\phi_{\mathrm{F}}(t)-\phi_{\mathrm{w}}(t)$.

Figure 9 shows the time-averaged phase difference $\phi_{\mathrm{d}}$ as a function of the dimensionless flow velocity for flags S1 to S4 listed in Table 2. In the all cases, the time-averaged phase difference never crosses $90^{\circ}$ and remains bounded in the $\left[0^{\circ} 50^{\circ}\right]$ range over the large-amplitude flapping regime. The different values of the phase difference may well be due to the effect of different structural damping for the different flags. (Similar observations have been made by Seyed-Aghazadeh et al. [12] for triangular prisms in cross-flow, who reported that no jump occurred from $\sim 0$ to $\sim 180^{\circ}$ in phase difference between flow forces and body motion, hence concluding that the oscillation was of the galloping type.)

The above observations suggest that flag motion and vortex shedding influence each other reciprocally; 


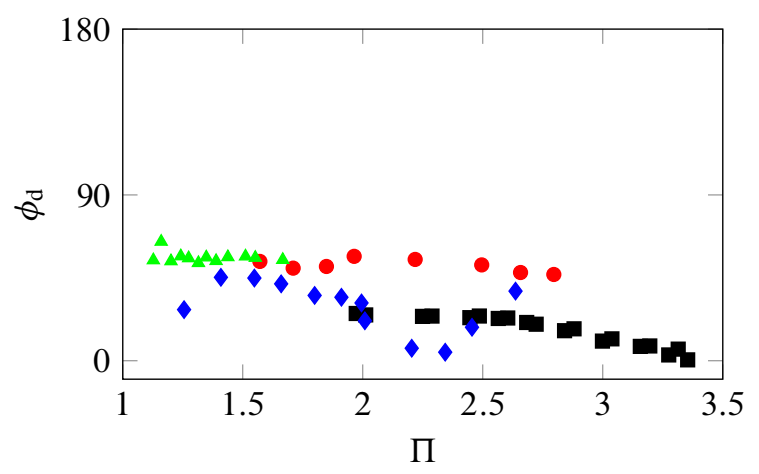

Figure 9: Time-averaged phase difference between transverse force (lift) and transverse displacement for [ם]

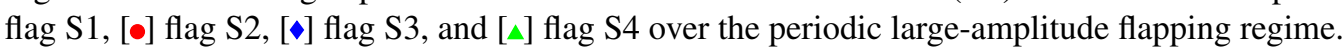

however, vortex shedding does not appear to be the cause for flapping of, at least, heavy inverted flags. Instead, the large-amplitude flapping of heavy inverted flags accompanied by high-frequency vortex shedding suggests that self-excited vibration may be the underlying mechanism, meaning that the timeaveraged movement-induced aerodynamic forces govern the motion. A similar conclusion has been reached for slender prismatic bodies with bluff cross-section and sufficiently long afterbody in cross-flow by Nemes et al. [8], Zhao et al. [17] and Seyed-Aghazadeh et al. [12], among others. The present observations also agree well with the computational predictions of Goza et al. [1], who report that, for heavy flags, the dominant frequency of lift (corresponding to the highest peak in the PSD plot) is greater than that of the tip displacement, suggesting that the motion is not "classical VIV".

Finally, it is recalled that according to a well-known classification by Naudascher and Rockwell [7], flow-induced vibrations are divided into three types: (i) extraneously induced excitation, (ii) instabilityinduced excitation, and (iii) movement-induced excitation (or self-excited vibration). Turbulence buffeting and vortex-induced vibration are classical examples of the first and second types, respectively, while flutter of an aircraft wing and of a cantilevered pipe conveying fluid are examples of the last type. If the flapping of heavy inverted flags is not an extraneously induced excitation (since the turbulence intensity was kept very low in our wind tunnel experiments) or an instability-induced excitation (as argued above); then, by induction, it has to be a movement-induced excitation.

\section{Conclusion}

Some experiments were described in this paper, aiming to examine the global dynamics of heavy inverted flags and, in particular, to evaluate the impact of periodic vortex shedding from the leading and trailing edges thereon.

The effects of adding serrations to the leading edge and a long rigid splitter plate to the trailing edge were investigated: only minor changes in the critical flow velocity, amplitude and frequency of oscillations resulted thereby. The overall dynamics and its features remained unchanged, and large-amplitude flapping was observed for all flags tested in the experiments, with or without the serrations and the splitter plate. With the splitter plate, TEVs are formed and shed from the free end of the plate (that is far from the flag), the effect of which on the flow field close to the flag is minimal; also, interactions between the two counter-rotating LEVs (if they are not completely disrupted by serrations) are inhibited by the splitter plate.

Force measurements provided some insight into the relationship between vortex shedding and largeamplitude flapping; a difference between the dominant (peak) frequencies of the lift and flapping was found in some cases. Moreover, it was shown that for heavier inverted flags, additional peaks, with power as great as or larger than that matching the dominant motion frequency, appear in the lift frequency spectrum. In addition, the lift and tip displacement were found to be desynchronised in the chaotic-like flow regime.

The experimental results presented in $\S \S 2.1-2.3$ suggest that self-excited vibration via a fluidelastic instability may be the underlying mechanism for the flapping motion of heavy inverted flags. The nearly- 
identical qualitative behaviour of plain inverted flags and those with a serrated leading edge and a splitter plate at the trailing edge suggests that the global (qualitative) dynamical characteristics of heavy inverted flags are not governed by the unsteady vortex shedding from the leading and/or trailing edges. In other words, periodic vortex shedding is not the cause, but an effect of large-amplitude flapping.

It is stressed that no flow visualization was carried out in the experiments with serrated flags and the splitter plate, and thus no information can be provided regarding the vortex formation and shedding from the leading and trailing edges. Nevertheless, PIV measurements conducted by Pazhani [9] showed that complex three-dimensional flow near the serrations disrupts regular vortex formation and shedding from the leading-edge-LEV become more stable and their periodic formation and shedding is delayed.

There are definitely potential correlations, yet to be discovered, between the phase dynamics and the underlying mechanism for large-amplitude flapping. Further investigations would be desirable to better clarify the distinction between VIV and the underlying mechanism for large-amplitude flapping of heavy flags.

Acknowledgments The authors gratefully acknowledge the financial support by the Natural Sciences and Engineering Research Council of Canada, the Solution Mining Research Institute and Pipeline Research Council International. It is a pleasure to acknowledge the benefit of insightful comments by Professor John E. Sader.

Declaration of Interests The authors report no conflict of interest.

\section{Bibliography}

[1] Andres Goza, Tim Colonius, and John Sader. Global modes and nonlinear analysis of inverted-flag flapping. Journal of Fluid Mechanics, 857:312-344, 2018. doi:10.1017/jfm.2018.728. ə arXiv:1709.09745.

[2] Pardha Gurugubelli and Rajeev Jaiman. Self-induced flapping dynamics of a flexible inverted foil in a uniform flow. Journal of Fluid Mechanics, 781:657-694, 2015. doi:10.1017/jfm.2015.515. ə oai:hal-03000231.

[3] Pardha Gurugubelli and Rajeev Jaiman. Large amplitude flapping of an inverted elastic foil in uniform flow with spanwise periodicity. Journal of Fluids and Structures, 90:139-163, 2019. doi:10.1016/j.jfluidstructs.2019.05.009. ə arXiv:1711.01065.

[4] Asif Khalak and Charles Williamson. Motions, forces and mode transitions in vortex-induced vibrations at low mass-damping. Journal of Fluids and Structures, 13(7-8):813-851, 1999. doi:10.1006/jfls.1999.0236. ๖ oai:hal-03000247.

[5] Daegyoum Kim, Julia Cossé, Cecilia Huertas Cerdeira, and Morteza Gharib. Flapping dynamics of an inverted flag. Journal of Fluid Mechanics, 736:R1-12, 2013. doi:10.1017/jfm.2013.555. ə Caltech:20131216092317508.

[6] Efstathios Konstantinidis, Jisheng Zhao, Justin Leontini, David Lo Jacono, and John Sheridan. Phase dynamics of effective drag and lift in vortex-induced vibration at low mass-damping. Journal of Fluids and Structures, 96, 2020. doi:10.1016/j.jfluidstructs.2020.103028. ə arXiv:1906.07375.

[7] Eduard Naudascher and Donald Rockwell. Flow-Induced Vibrations: An Engineering Guide. Rotterdam: A.A. Balkema, 1994.

[8] András Nemes, Jisheng Zhao, David Lo Jacono, and John Sheridan. The interaction between flow-induced vibration mechanisms of a square cylinder with varying angles of attack. Journal of Fluid Mechanics, 710: 102-130, 2012. doi:10.1017/jfm.2012.353. ə oai:hal-00752464.

[9] Kaushik Murugesan Pazhani. An experimental investigation of the dynamics of an inverted serrated flag. Master's thesis, Illinois Institute of Technology, 2018. hdl:10560/islandora:1001299.

[10] Jaeha Ryu, Sung Goon Park, Boyoung Kim, and Hyung Jin Sung. Flapping dynamics of an inverted flag in a uniform flow. Journal of Fluids and Structures, 57:159-169, 2015. doi:10.1016/j.jfluidstructs.2015.06.006. ə oai:hal-03000240.

[11] John Sader, Julia Cossé, Daegyoum Kim, Boyu Fan, and Morteza Gharib. Large-amplitude flapping of an inverted flag in a uniform steady flow - a vortex-induced vibration. Journal of Fluid Mechanics, 793:524-555, 2016. doi:10.1017/jfm.2016.139. ə oai:hal-03000258.

[12] Banafsheh Seyed-Aghazadeh, Daniel Carlson, and Yahya Modarres-Sadeghi. Vortex-induced vibration and galloping of prisms with triangular cross-sections. Journal of Fluid Mechanics, 817:590-618, 2017. doi:10.1017/jfm.2017.119. ə oai:hal-03000265.

[13] Mohammad Tavallaeinejad. Nonlinear Dynamics of Inverted Flags: A Theoretical and Experimental Investigation. PhD thesis, McGill University, 2020. ə [pdf]. 
[14] Mohammad Tavallaeinejad, Mathias Legrand, and Michael Païdoussis. Nonlinear dynamics of slender inverted flags in uniform steady flows. Journal of Sound and Vibration, 467:115048, 2020. ISSN 0022-460X. doi:10.1016/j.jsv.2019.115048. ə oai:hal-02342918.

[15] Mohammad Tavallaeinejad, Michael Païdoussis, Mathias Legrand, and Mojtaba Kheiri. Instability and the post-critical behaviour of two-dimensional inverted flags in axial flow. Journal of Fluid Mechanics, 890:A14, 2020. doi:10.1017/jfm.2020.111. ə oai:hal-02520870.

[16] Yuelong Yu, Yingzheng Liu, and Yujia Chen. Vortex dynamics behind a self-oscillating inverted flag placed in a channel flow: Time-resolved particle image velocimetry measurements. Physics of Fluids, 29(12): 125104(1-15), 2017. doi:10.1063/1.5001967. ə oai:hal-03000272.

[17] Jisheng Zhao, Justin Leontini, David Lo Jacono, and John Sheridan. Fluid-structure interaction of a square cylinder at different angles of attack. Journal of Fluid Mechanics, 747:688-721, 2014. doi:10.1017/jfm.2014.167. ə hdl:1959.3/378617. 